\title{
efectos del recubrimiento de los cables sobre el comportamiento de las vigas pretensadas
}

PAUL H. KAAR y DONALD D. MAGURA, ingenieros

\section{Sîneopeniss}

Se trata de un estudio sobre el revestimiento con tubos de plástico, usado para prevenir la adherencia entre el cable y el hormigón cerca de los extremos de las vigas pretensadas. Ensayos realizados sobre cinco vigas $\mathrm{T}$ de $10,3 \mathrm{~m}$ de longitud indicaron que la longitud de empotramiento que exige la sección 2611 de la 1963 ACI Building Code es inadecuada en el caso de cables revestidos. La longitud necesaria para que se desarrollen la flexión máxima y el esfuerzo cortante en las vigas, cuando se usa el revestimiento, es doble de la longitud del anclaje que señala dicha norma.

\section{Introducción}

Dos son los métodos que se emplean para limitar los esfuerzos de com. presión y tracción en el hormigón cerca de los extremos de los elementos de hormigón pretensado: 1) Algunas de las armaduras pretensadas son curvadas hacia arriba, cerca de los extremos de los elementos (procedimiento de fabricación un poco costoso). 2) La adherencia al hormigón puede evitarse en algunas de las armaduras pretensadas en las zonas de los extremos. Una de las formas de evitar la adherencia es mediante el empleo del tubo de plástico, método generalmente conocido con el nombre de «blanketing».

El objeto de la investigación descrita aquí era estudiar los posibles efectos del revestimiento sobre el comportamiento de una viga a flexión con cargas de servicio y sobre la flexión final, adherencia y esfuerzo cortante de las vigas pretensadas.

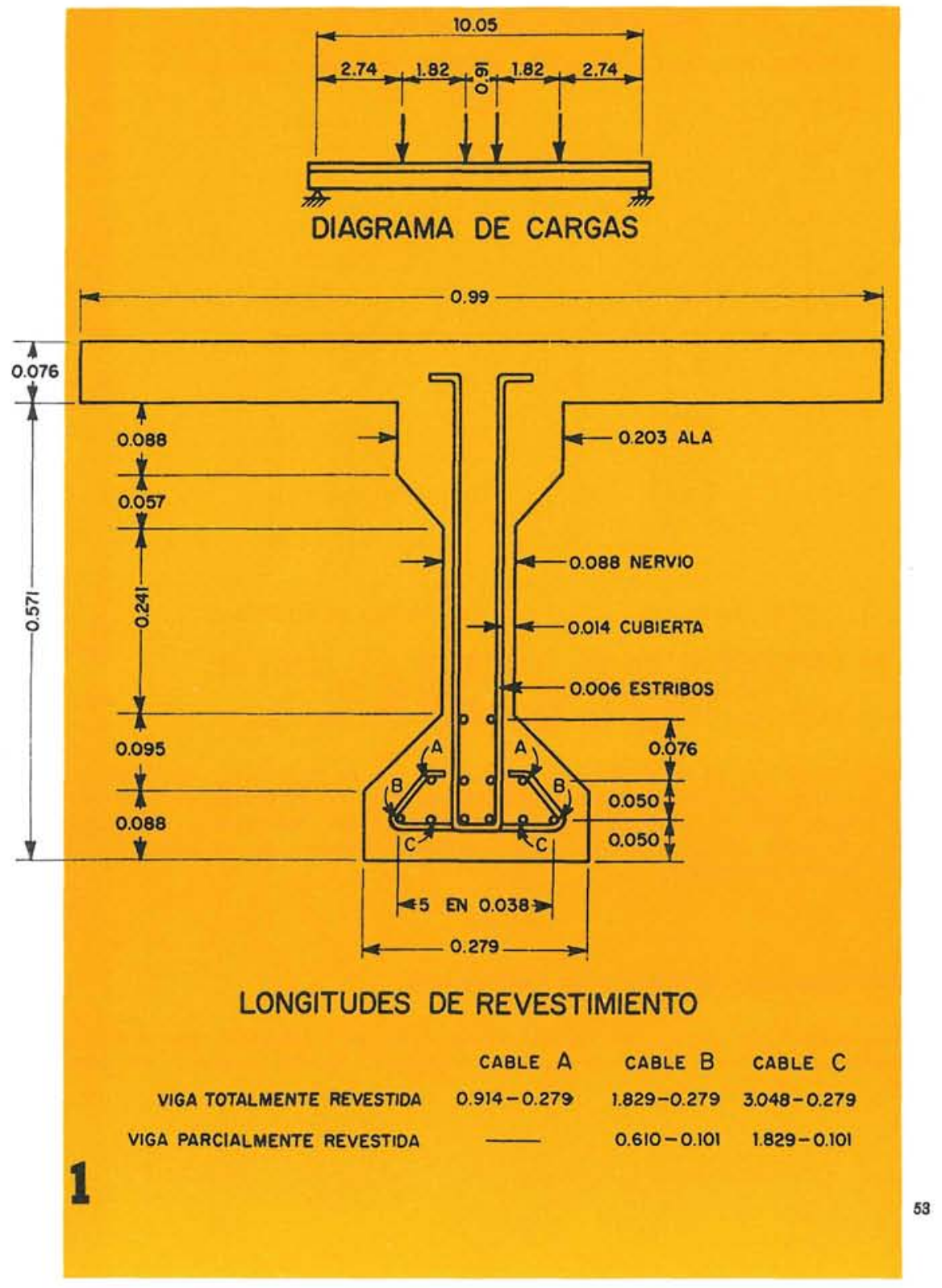


TENSION DEL CABLE $\left(12.304 \mathrm{~kg} / \mathrm{cm}^{2}\right)$ TENSION DEL CABLE $\left(9.843 \mathrm{~kg} / \mathrm{cm}^{2}\right)$

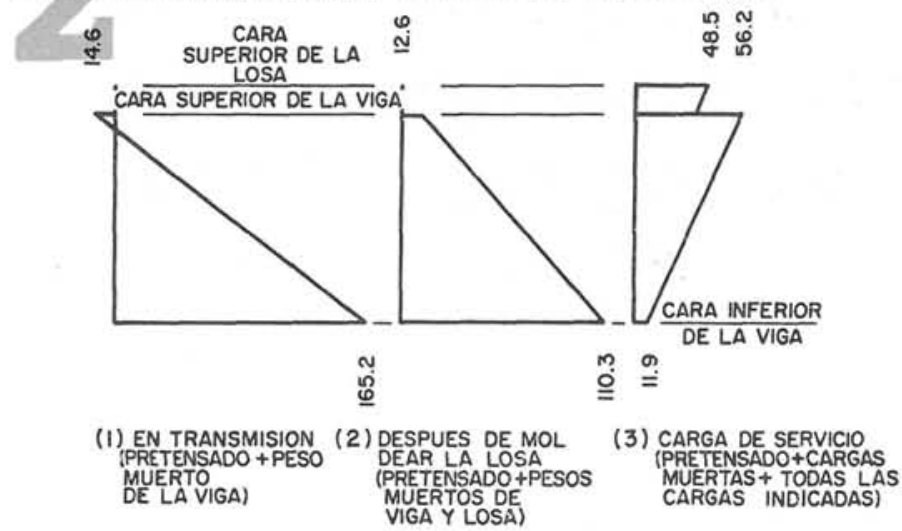

\section{(a) ESFUERZOS EN LA SECCION DE LUZ MEDIA DE TODAS LAS VIGAS}

ESFUERZO EN EL CABLE $\left(12.304 \mathrm{~kg} / \mathrm{cm}^{2}\right)$ ESFUERZO EN EL CABLE $\left(9.843 \mathrm{~kg} / \mathrm{cm}^{3}\right)$

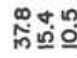
ํํำ

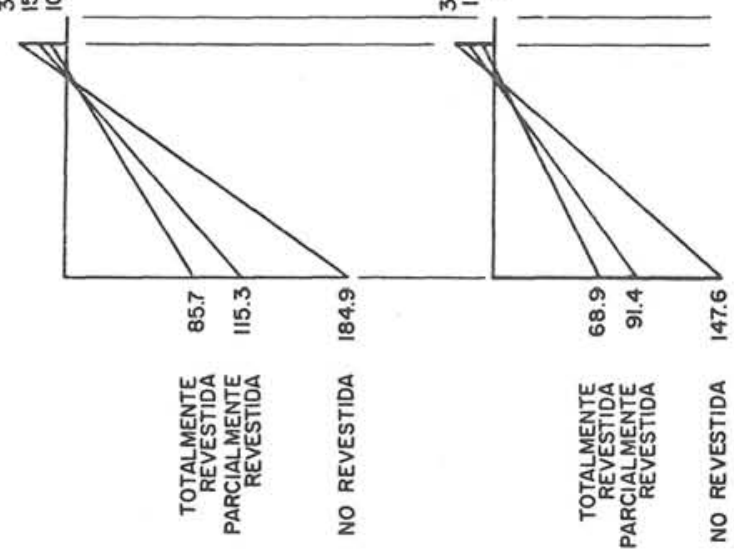

(1) EN TRANSMISION

(2) DESPUES DE PERDIDAS

\section{(b) ESFUERZOS EN EL LIMITE DE LA ZONA DE TRANSMISION DE PRETENSADO $660 \mathrm{~mm}$ DESDE EL EXTREMO DE LA VIGA}

\section{Fondo y alcance \\ Misión de la adherencia}

La misión inicial de la adherencia es transmitir la tensión debida al pretensado de las armaduras al elemento de hormigón. Los estudios en laboratorio sobre la longitud adherida de cable hacían necesaria para tal transmisión una serie de datos previos (1); también son útiles otros numerosos documentos que tratan sobre la adherencia en hormigón pretensado. Bajo cargas de servicio, el esfuerzo en la armadura pretensada continúa generalmente próximo al nivel de pretensado eficaz. La integridad estructural del elemento sigue dependiendo en gran parte de la adherencia en las regiones de transferencia del esfuerzo, y los esfuerzos de adherencia en flexión son despreciables.

Para cargas comprendidas desde la carga de servicio hasta la carga de flexión final, sin embargo, el esfuerzo en las armaduras pretensadas debe eventualmente aumentar considerablemente sobre el nivel de pretensado. Trabajos de laboratorio previos (2) han demostrado que una considerable longitud de empotramiento adherida para transmitir el esfuerzo más allá de dicha longitud es adecuada para transmitir estos esfuerzos aumentados al acero.

Si se habilita una longitud de empotramiento inadecuada, el esfuerzo final está determinado más bien por la adherencia que por la flexión. Hay tres casos de pérdida de adherencia en los cables: 1) $\mathrm{La}$ pérdida progresiva de adherencia comienza en las grietas de flexión; 2) La pérdida de adherencia general se inicia a lo largo de toda la longitud de empotramiento; 3) La trabazón mecánica entre la superficie helicoidal del cable y el hormigón se destruye.

Hay que advertir que la trabazón mecánica es suficiente para mantener un esfuerzo considerable en el cable después de producirse una gran pérdida de adherencia. En muchos casos, la tensión del cable después de una pérdida de adherencia general desciende sólo hacia el nivel de pretensado y no hacia cero, como se podría temer. Así, el efecto final de una longitud de empotramiento inadecuada podría ser una pérdida prematura de flexión en un esfuerzo reducido en el cable, correspondiente a un momento flector final menor que el último esfuerzo de flexión calculado.

\section{Solicitaciones debidas a la adherencia}

La sección 2611 de la 1963 ACI Building Code, ACI 318-63 (3), determina para un empotramiento de cables pretensados adheridos desde la sección considerada que la distancia, en milímetros, no debe ser menor que:

$$
\left(f_{s u}-\frac{2}{3} f_{s e}\right) D \text {, }
$$


en donde:

$$
\begin{aligned}
D= & \text { diámetro teórico del cable, en } \\
& \text { milímetros; } \\
f_{s u}= & \text { esfuerzo calculado en acero pre- } \\
& \text { tensado bajo carga final, en ki- } \\
& \text { logramos } / \mathrm{cm}^{2} ; \\
f_{s e}= & \text { esfuerzo real del acero después } \\
& \text { de las pérdidas, en } \mathrm{kg} / \mathrm{cm}^{2} .
\end{aligned}
$$

La longitud de empotramiento requerida por la ecuación [1], se basa en ensayos de vigas con todos los cables adheridos desde la sección del momento máximo hasta el extremo de las vigas. El extremo de la longitud de empotramiento puede entonces solapar el esfuerzo que transmite cerca de los soportes de la viga, donde existe un estado de precompresión flexional incluso para cargas altas y donde la reacción vertical en el soporte de la viga suple una compresión lateral. Sin embargo, cuando los cables están revestidos en una distancia considerable dentro del elemento, el esfuerzo transmitido y la adherencia del empotramiento en flexión pueden situarse en una región concreta sometida a tensión y se alcanza antes la carga final, incluso en rotura. Bajo estas severas condiciones, la longitud de empotramiento dada por la fórmula [1] puede ser insuficiente.

\section{Alcance de los experimentos}

La investigación fue realizada al valorar, separadamente, los efectos del revestimiento en comportamiento a flexión y en capacidad a esfuerzo cortante.

Se eligieron y ensayaron tres vigas en el estudio del comportamiento a flexión. La viga 1 no tenía cables revestidos y se empleó como elemento de comparación. La viga $\mathbf{2}$, designada como "parcialmente revestida", tenía cables tan revestidos que las longitudes de empotramiento fueron dobles que las obtenidas por la expresión [1] La viga "totalmente revestida", viga 3 , se dispuso con un empotramiento de los cables revestidos igual a las longitudes obtenidas por [1]. Las tres vigas fueron super-reforzadas por medio de estribos para evitar interferencias

Fig. 3. Indicación del cierre del revestimiento del cable.

Fig. 4. Ensayo de viga bajo carga cíclica.

Fig. 5. Ensayo de viga bajo carga estática.
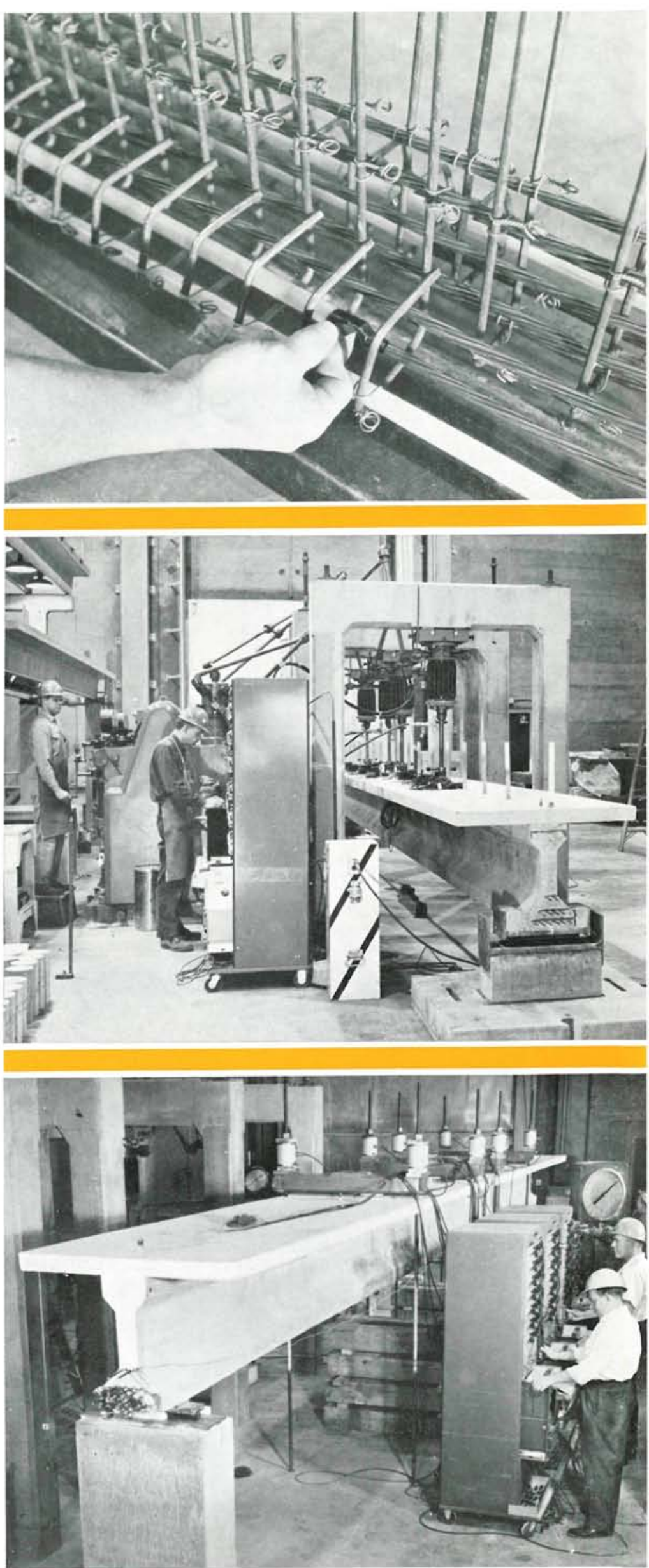
entre los comportamientos relativos a la adherencia, el esfuerzo cortante y la flexión. Las vigas fueron sometidas a $5 \times 10^{6}$ ciclos bajo carga de servicio y a continuación ensayadas estáticamente a rotura.

La investigación del cortante implicó el ensayo estático de destrucción de una viga no revestida, viga 4, y una viga con cables parcialmente revestidos, viga 5. Las vigas eran semejantes a aquellas en que se verificó el estudio a flexión, excepto que el número de estribos se redujo en una proporción que algunos efectos de la capacidad a esfuerzo cortante de los cables revestidos habían probado. Los estribos en las vigas 4 y 5 estaban separados vez y media de la indicada por la sección 2610 de la 1963 ACI Building Code, suponiendo una fuerza concreta de $351,5 \mathrm{~kg} / \mathrm{cm}^{2}$. Hay que hacer notar que la viga 5 tenía un refuerzo de nervio ligeramente más grueso que la viga 4 , debido a su menor pretensado en las zonas revestidas.

\section{Ensayos de vigas}

Todas las vigas ensayadas presentaban la sección transversal indicada en la figura 1. El premoldeado sección I es un modelo tipo III AASHO-PCI para viga de puente de hormigón pretensado a escala $1 / 2$. Las vigas tenían $10,3 \mathrm{~m}$ de longitud, simplemente apoyadas sobre una luz de $10,0 \mathrm{~m}$. Después de haber elegido la sección transversal de la viga y la luz, el objeto consistía en utilizar la viga para calcular el esfuerzo de pretensado y su localización para utilizar los esfuerzos admisibles dados en la tabla 1.

Doce cables de siete alambres y un diámetro de $9,5 \mathrm{~mm}$ se colocaron como indica la figura 1. El proyecto admitía que el cable debía tensarse inicialmente a 12.304 $\mathrm{kg} / \mathrm{cm}^{2}$ y que la tensión después de todas las pérdidas debería ser de $9.843 \mathrm{~kg} / \mathrm{cm}^{2}$.

El valor del momento flector final en la viga estaba calculado según las ecuaciones (26-4) y (26-6) de la sección 2608 de la

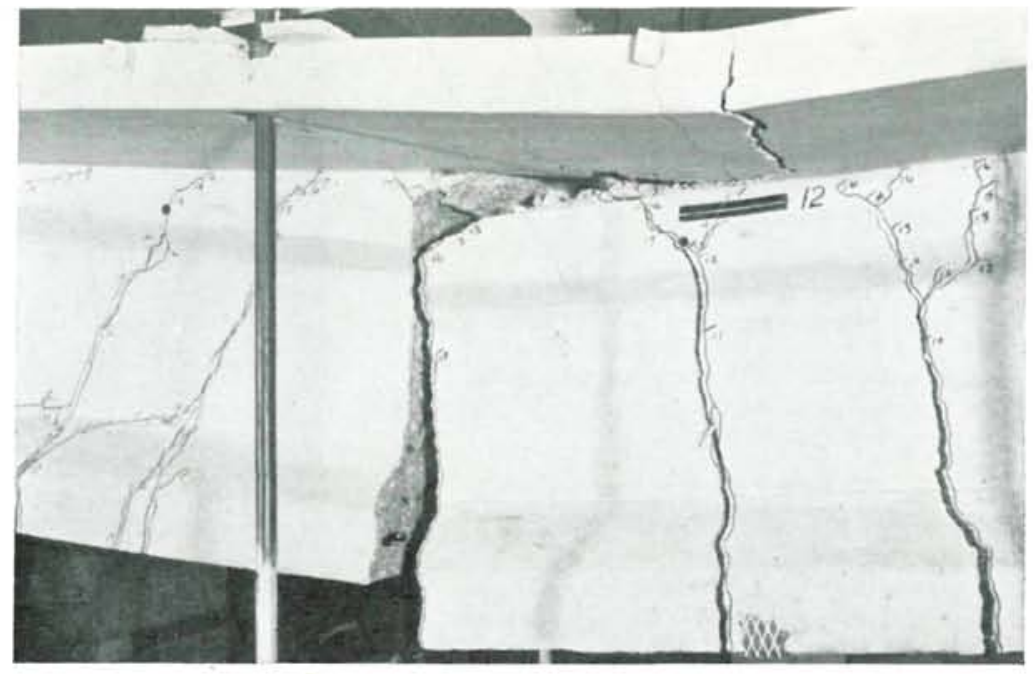

Fig. 6. Area de fractura: Viga 1.

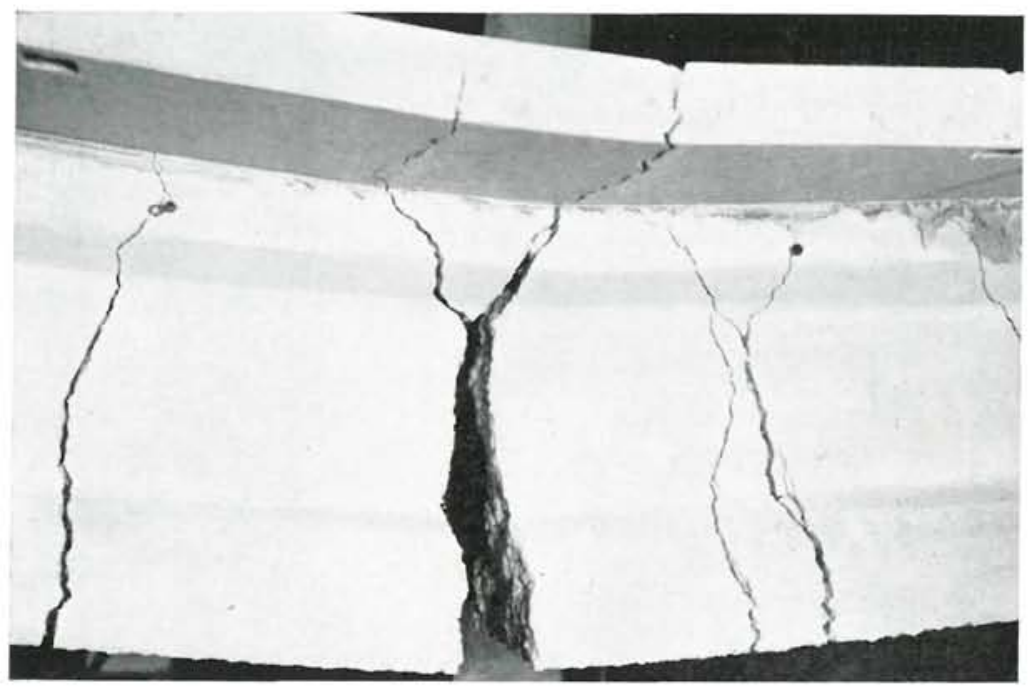

Fig. 7. Area de fractura: Viga $\mathbf{z}$.

Fig. 8. Area de fractura: Viga $\mathbf{3}$.

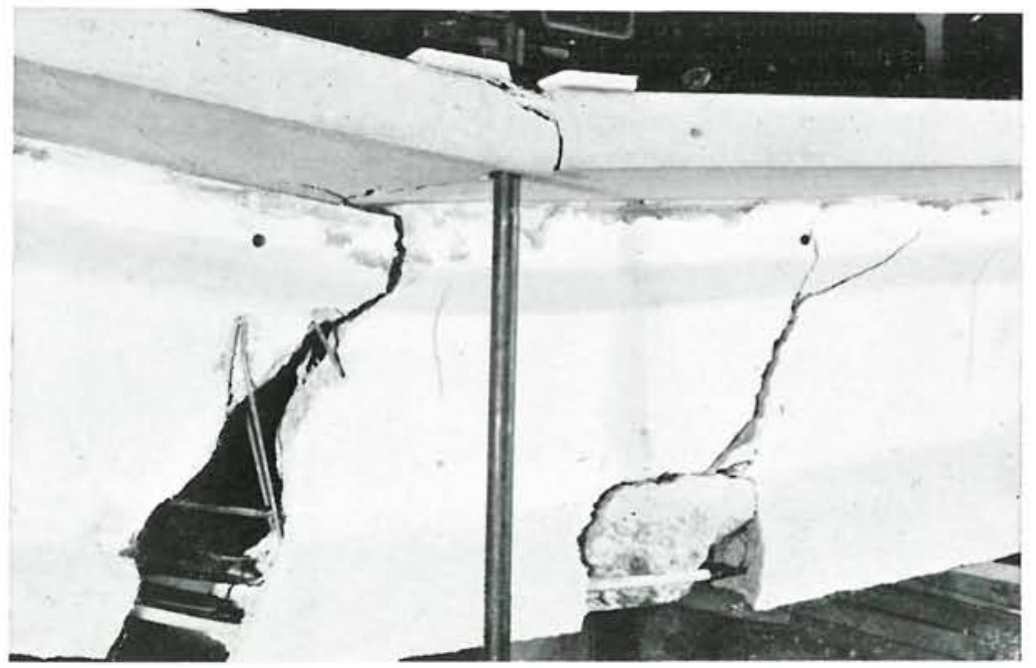


1963 ACI Code, resistiendo el cable un esfuerzo final, $f^{\prime}$, de 17.577 kilogramos $/ \mathrm{cm}^{2}$. El momento de la carga de servicio estaba calculado por el requisito de la sección 1.13 .6 de la 1961 AASHO Standard Specifications for Highway Bridges (4), según el cual cada sección de la viga tiene una fuerza final de $(1,5 D+$ $+2,5 L)$ al menos, donde $D$ es el efecto del peso muerto y $L$ el de la llamada carga viva, incluyendo el choque. La magnitud y localización de las cargas vivas estaban impuestas por el momento de la carga de servicio, con el requisito adicional de que el diagrama de momentos debería ser semejante a un diagrama racional para vigas de puente $o$ carretera. La carga viva consistía en cuatro cargas concentradas iguales, distribuidas como indica la figura 1 .

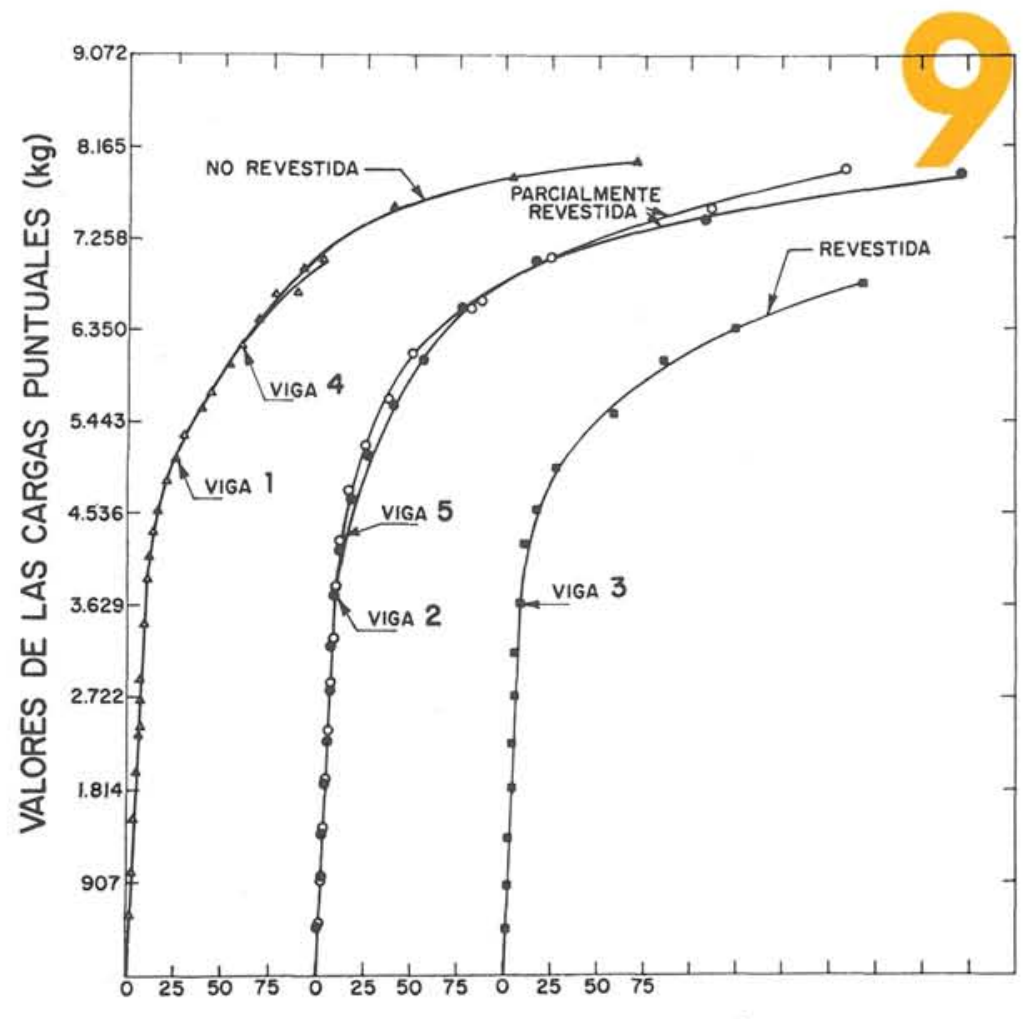

CENTRO DE LA FLECHA (mm)

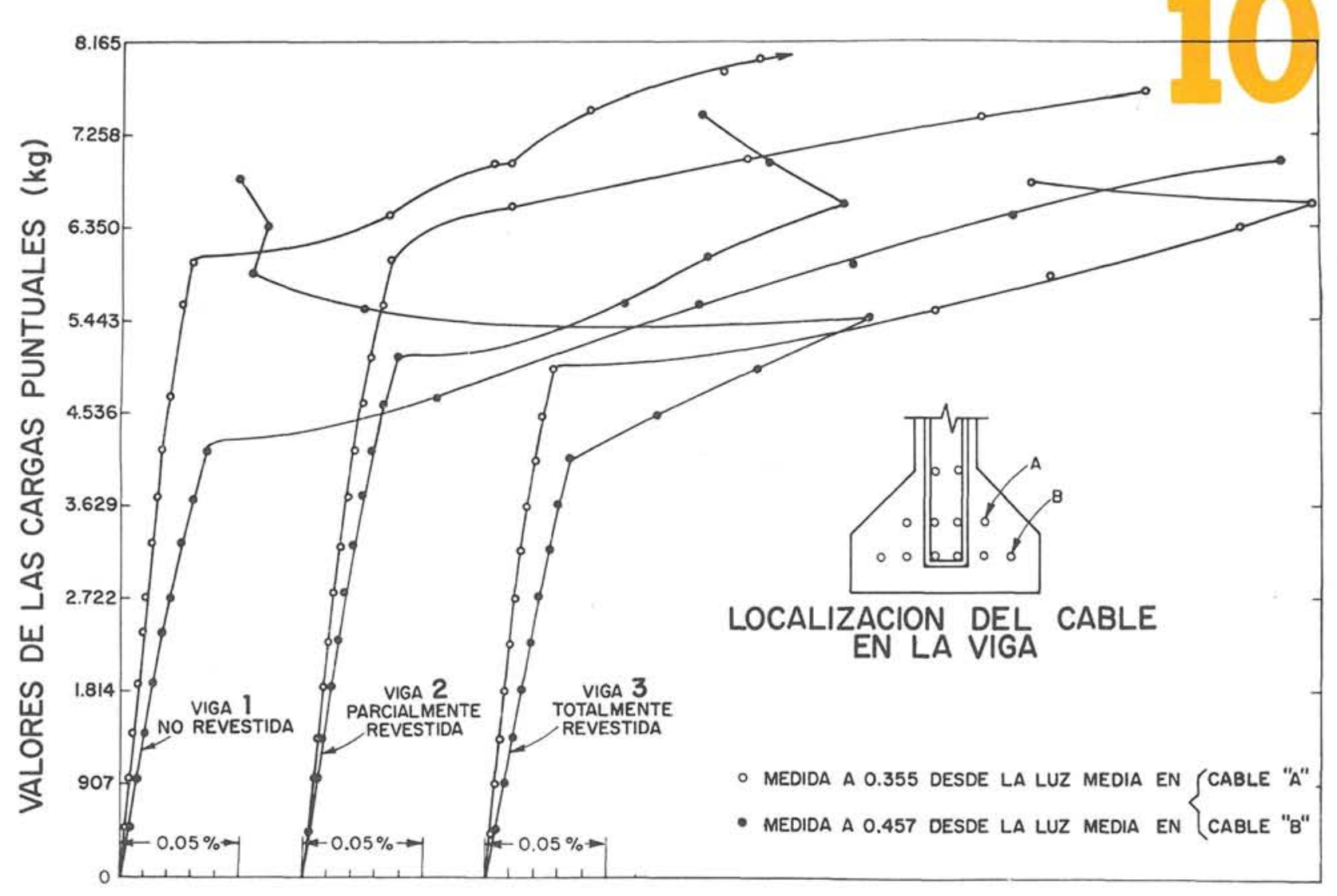

TENSION DEL CABLE SUPERIOR A LA DE PRETENSADO 
El diagrama de momentos en rotura debido a las cargas estaba calculado suponiendo que el momento máximo era igual al momento final en la sección transversal de la viga no revestida. De este diagrama estaban determinados los traza. dos en los que era posible reducir la cantidad de refuerzos de tensión por revestimiento de los cables. La posición de los cables revestidos en la sección transversal de la viga y las longitudes de recubrimiento de los extremos de las vigas están dadas en la figura 1.

Los esfuerzos en el hormigón durante el pretensado inicial y en otros momentos del desarrollo de las cargas están dados en la figura 2. Los esfuerzos finales están calculados para un límite inferior de $660,4 \mathrm{~mm}$ de la zona de transmisión de pretensado. Esta longitud de transferencia estaba determinada por un trabajo previo (1).

\section{Fabricación - instrumentación}

Las vigas están fabricadas y ensayadas con métodos comúnmente empleados en el PCA Structural Laboratory. Una descripción general de ayudas y métodos de ensayo se da en (5).

\section{Fabricación}

Las vigas premoldeadas estaban fabricadas en un lecho pretensado elevado sobre la planta del laboratorio de ensayo. Los cables fueron tesados individualmente por medio de un gato de $508,0 \mathrm{~mm}$ de carrera. $\mathrm{La}$ tensión en el cable se calculó por un medidor de cargas colocado entre la mordaza del cable y la placa de sujeción en el extremo muerto del lecho. Los $12.304 \mathrm{~kg} / \mathrm{cm}^{2}$ de tensión del cable se consiguieron sobretesándolo inicialmente de manera que las pérdidas no le hicieran decrecer por debajo del valor considerado. $\mathrm{La}$ variación en las mediciones del cable después de relajar el esfuerzo, indicó que $9.843 \mathrm{~kg} / \mathrm{cm}^{2}$, teniendo en cuenta las pérdidas, es una cifra realista.

Terminada la colocación de los estribos, se vertió el hormigón al día siguiente de haber tesado los cables. Se interrumpió el curado húmedo de las vigas al cabo de 3 días y se cortaron los cables con un soplete de acetileno.

El encofrado de las losas de cubierta estaba volado por ambos lados de la viga premoldeada, de tal forma que el peso muerto de la losa, juntamente con los moldes estaba sostenido por la viga interior como un tramo simple. De esta manera se simulaba el método de práctica habitual. La losa de cubierta estuvo en curado hú- 


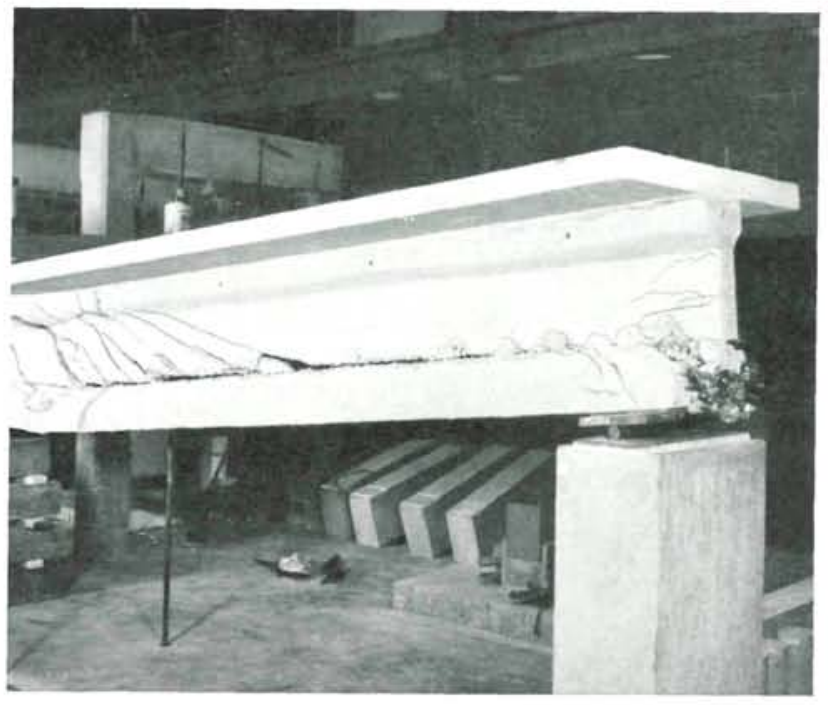

Fig. 12. Area de fractura: Viga $\mathbf{4}$.

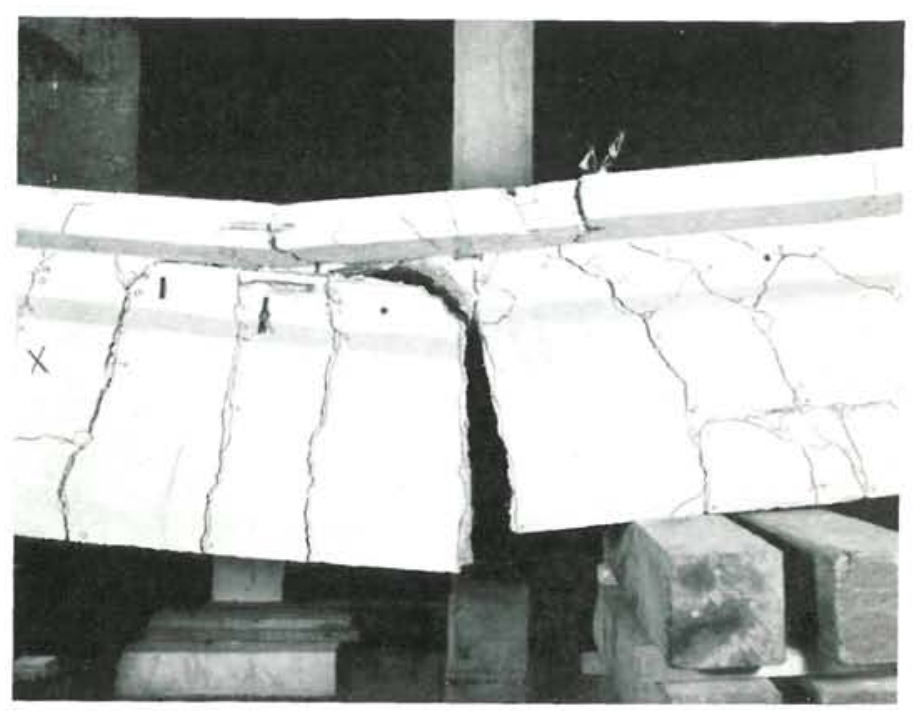

Fig. 13. Area de fractura: Viga $\mathbf{5}$.

medo durante 3 días después del moldeado y las muestras tipo estuvieron expuestas luego a la atmósfera del laboratorio a $21,11^{\circ} \mathrm{C}$ y un porcentaje de humedad relativa del 50 por 100.

TABLA 1. LIMITACIONES DE ESFUERZOS

Valores standard AASHO para carreteras y puentes, 1961

\begin{tabular}{c|l|c}
\hline \multicolumn{1}{c|}{ ET A P A } & \multicolumn{1}{|c}{ ESFUER Z } & LIMITE \\
\hline $\begin{array}{l}\text { En el momento de la trans- } \\
\text { ferencia } \\
f_{c i}^{\prime}=281,2 \mathrm{~kg} / \mathrm{cm}^{2}\end{array}$ & Compresión & $06 f_{c i}^{\prime}=168,7 \mathrm{~kg} / \mathrm{cm}^{2}$ \\
\hline Para carga total calculada & $\begin{array}{l}\text { Compresión } \\
\text { Tracción en la cara inferior } \\
f_{c}^{\prime}=351,5 \mathrm{~kg} / \mathrm{cm}^{2}\end{array}$ & $3 \sqrt{f_{c i}^{\prime}}=50 \mathrm{~kg} / \mathrm{cm}^{2}$ \\
\hline
\end{tabular}

\section{Mareriales}

El acero pretensado empleado era cable de siete alambres, con un diámetro de $9,5 \mathrm{~mm}$ y un área de $0,51 \mathrm{~cm}^{2}$. Todo el cable estaba desprovisto de herrumbre y se limpió la superficie con aceite antes de tesarlo. Las propiedades del cable están indicadas en la tabla 2.

Los estribos empleados en las vigas 1, 2 y 3 eran barras lisas del número 2. Los estribos de las vigas 4 y 5 eran barras deformadas del número 2 y estaban elegidas entre aquellas cuyo rendimiento estuviera comprendido entre $3.550,5$ y $3.831,7 \mathrm{~kg} / \mathrm{cm}^{2}$.

El hormigón empleado en la fabricación de las vigas pretensadas consistía en una mezcla de cemento portland tipo III y áridos de $9,5 \mathrm{~mm}$ de tamaño máximo. En las losas de cubierta se empleó una mezcla de ce- 
TABLA 2. PROPIEDADES DE LOS CABLES PRETENSADOS

\begin{tabular}{c|c|c}
\hline $\begin{array}{c}\text { Viga } \\
\text { número }\end{array}$ & $\begin{array}{c}\text { Esfuerzos producidos } \\
\left(\mathrm{kg} / \mathrm{cm}^{2}\right)\end{array}$ & $\begin{array}{c}\text { Esfuerzos de tensión } \\
\left(\mathrm{kg} / \mathrm{cm}^{2}\right)\end{array}$ \\
\hline $\mathbf{1}$ & & 19.475 \\
$\mathbf{2}$ & 18.702 & 19.475 \\
$\mathbf{3}$ & 18.702 & 19.475 \\
$\mathbf{4}$ & 18.702 & 19.616 \\
$\mathbf{5}$ & 18.983 & 19.124 \\
\hline
\end{tabular}

- Esfuerzo en \% compensado.

mento portland tipo I. En ambos casos; el hormigón tenía aire ocluido. Los esfuerzos en las probetas de hormigón dados en la tabla 3 se basan en pruebas realizadas sobre cilindros, de $152,4 \times 304,8$ mm, tomados de amasadas alternadas. Los cilindros fueron curados y almacenados con la viga y las losas de cubierta. Todos los esfuerzos que se concretan para las vigas 4 y $\mathbf{5}$ están dados en el momento del ensayo a rotura.

Los revestimientos que cubren el cable eran de tubo flexible de polietileno, de 11,11 mm de diámetro interno y $1,58 \mathrm{~mm}$ de espesor de pared. Los extremos de los tubos que cubren los cables estaban cerrados para evitar la entrada del cemento. La figura 3 enseña la cobertura de los cables y el cierre de los extremos.

\section{Instrumental}

Las tensiones fueron calculadas por medio de una resistencia eléctrica SR-4, que mide la tensión por medio de un ábaco de contadores en línea. Los medidores del cable se hallaban alineados a lo largo de una hélice en el cable. En todas las vigas, varios cables en el fondo del lecho estaban arqueados 0,45 metros y $1,67 \mathrm{~m}$ a uno y otro lado del eje. Por añadidura, la tongada intermedia de refuerzo en las vi-

TABLA 3. PROPIEDADES DEL HORMIGON

\begin{tabular}{|c|c|c|c|c|}
\hline \multirow{3}{*}{$\begin{array}{l}\text { Viga } \\
\text { número }\end{array}$} & \multicolumn{4}{|c|}{ ESFUERZOS DE COMPRESION $\left(\mathrm{kg} / \mathrm{cm}^{2}\right)$} \\
\hline & \multicolumn{2}{|c|}{ Bajo efecto de carga cíclica } & \multicolumn{2}{|c|}{ En prueba estática de rotura } \\
\hline & Cubierta & Viga & Cubierta & Viga \\
\hline 1 & 284,7 & 589,2 & 374,0 & 651,0 \\
\hline 2 & 267,8 & 461,9 & 399,3 & 524,4 \\
\hline 3 & 319,1 & 549,8 & 393,7 & 578,6 \\
\hline \multirow{2}{*}{$\begin{array}{l}\text { Viga } \\
\text { número }\end{array}$} & \multicolumn{2}{|c|}{$\begin{array}{l}\text { Rotura de probeta } \\
\qquad\left(\mathrm{kg} / \mathrm{cm}^{2}\right)\end{array}$} & \multicolumn{2}{|c|}{$\begin{array}{l}\text { Esfuerzo de compresión } \\
\qquad\left(\mathrm{kg} / \mathrm{cm}^{2}\right)\end{array}$} \\
\hline & Cubierta & Viga & Cubierta & Viga \\
\hline 4 & 35,6 & 40,0 & 347,3 & 492,1 \\
\hline 5 & 39,7 & 46,1 & 345,2 & 557,5 \\
\hline
\end{tabular}


gas 1, 2 y 3 tenía arqueados 2,59 m desde la luz media. Los medidores en el tramo medio del hormigón estaban situados en la parte más alta, a un lado y $25,4 \mathrm{~mm}$ bajo la superficie y en la parte de la viga que trabaja a tracción. A un lado de la luz media en las vigas 4 y $\mathbf{5}$, los estribos entre el apoyo y el punto de carga exterior estaban arqueados en la fibra neutra de la sección compuesta.

Las flechas se midieron con una gran precisión enrasando las escalas unidas a la cubierta encima de los apoyos y en la luz media. Las vigas 4 y 5 también tenían escalas a $1 / 4$ de la luz y para las vigas 2 y 3 las escalas estaban situadas en intervalos aún más frecuentes a lo largo de la luz.

El deslizamiento relativo final entre cables y hormigón se calculó con medidores de cuadrante montados en los extremos salientes de los cables.

En los ensayos estáticos a rotura, la fuerza en cada punto de carga se midió con cargas fijas colocadas en el conjunto de cargas.

\section{Procedimientos de ensayo}

Las vigas 1,2 y 3 fueron sometidas a $5 \times 10^{6}$ ciclos en el estudio del ensayo de rotura estático de la carga viva anterior. La carga dinámica se aplicó por medio de cuatro pisones de un pulsador hidráulico unitario Amsler, situando las cargas como se indicaba en la figura 1. Una viga bajo carga cíclica está representada en la figura 4. Primero se cargó cada viga estáticamente con cinco series sucesivas de cargas vivas. Entre la primera y quinta series las cargas se aplicaron con un aumento gradual de $226,8 \mathrm{~kg}$ en cada punto de carga, y los esfuerzos y flechas se midieron para cada valor de las cargas. En seguida se cargó la viga dinámicamente, llevándose a cabo ensayos estáticos después de, aproximadamente, $1,2,5$ y $5 \times 10^{6}$ ciclos. Al completar los $5 \times 10^{6}$ ciclos, se cambió de sitio a la viga para ser ensayada estáticamente hasta su destrucción.

Todas las vigas se cargaron a rotura con el método de ensayo que se indica en la figura 5. El aparato de carga estaba colocado encima de las varillas y crucetas de acero, y las cargas se aplicaron por medio de martillos hidráulicos, reaccionando contra la cara inferior de la planta de ensayo (5). Las cuatro cargas concentradas iguales se aplicaron en incrementos de $453,6 \mathrm{~kg}$, leyéndose las tensiones, flechas y deslizamientos en los extremos obtenidos para cada valor de las cargas.

\section{Resultados del ensayo: Vigas 1,2 y $\mathbf{3}$ \\ Esfuerzos}

Los momentos finales medidos y calculados y otras características de las tres vigas se dan en la tabla 4. El momento final calculado estaba computado empleando la distribución del esfuerzo equivalente rectangular de la 1963 ACI Building Code. Después que las propiedades del hormigón y del acero fueron determinadas con todo cuidado y se aplicó la distribución de cargas con toda exactitud, se empleó un $\Phi$ unitario en la expresión:

$$
M_{u}=\Phi \cdot A_{s} \cdot f_{s u} \cdot d(1-0,59 q)
$$

en donde:

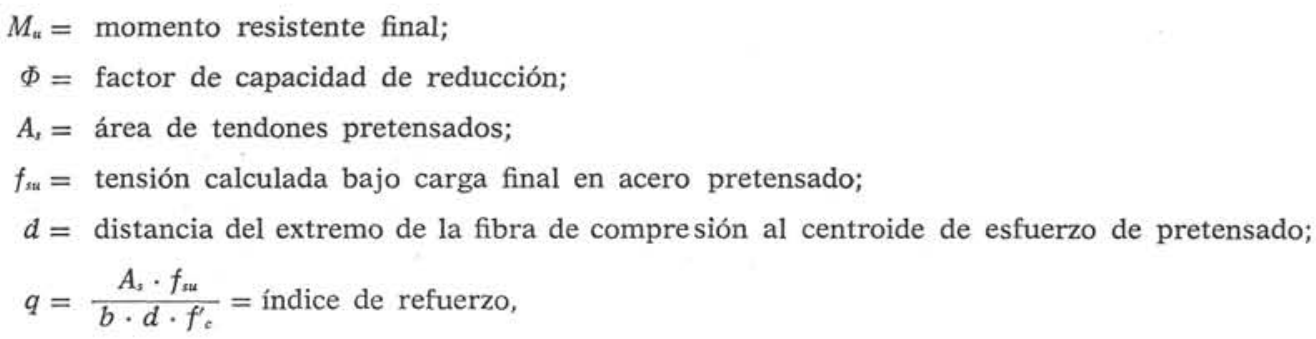


en la cual:

$$
\begin{aligned}
b & =\text { anchura de la cara de compresión del ele mento flexional; } \\
f^{\prime}{ }_{c} & =\text { esfuerzo de compresión del hormigón. }
\end{aligned}
$$

El cálculo del esfuerzo final estaba basado en un valor para $f_{s u}$ igual al esfuerzo final medido en los cables de siete hilos empleados.

En el caso de las vigas 1 y 2 todos los cables rompieron simultáneamente cerca de la carga final después de dar de sí considerablemente. Los esfuerzos en estas dos vigas fueron casi los mismos, siendo las relaciones de momento ensayado a momento calculado 0,98 y 0,96 , respectivamente.

La viga 3 , con cables totalmente revestidos, mostró una deficiencia de esfuerzo mucho mayor. La viga no rompió cerca del punto de momento máximo, sino en un punto de carga situado a $2,2 \mathrm{~m}$ de la luz media. Dos de los cables revestidos se extendían desde el extremo de la viga hasta una distancia de $2,0 \mathrm{~m}$ de la luz media, con lo que sólo actuaban en la sección de rotura diez cables. Las figuras 6, 7 y 8 indican las áreas de fractura de las tres vigas. El cable revestido puede verse sobresaliendo del hormigón en la viga de cables totalmente revestidos. Mientras el momento final ensayado era el 84 por 100 del momento teórico en el centro de la viga, el momento ensayado en el punto de fractura era sólo el 76 por 100 del momento final teórico en ese punto. Sin embargo, la viga sostuvo el 84 por 100 de la carga teórica, y por esta razón la relación del momento en la tabla 4 está registrada como 0,84 .

\section{Diagramas carga-flecha}

Las observaciones carga-flecha se tomaron en los ensayos estáticos para descubrir, si era posible, alguna pérdida de adherencia en los cables por efecto de la carga de servicio durante la actuación de los $5 \times 10^{6}$ ciclos. Los resultados de estas observaciones no indican la evidencia de un fallo en la adherencia para estos valores de carga. La relación carga-flecha era lineal, y en ninguna de las vigas ensayadas había una diferencia significativa entre la relación en la primera carga y la de después de los $5 \times 10^{6}$ ciclos.

Las curvas carga-flecha dadas en la figura 9, trazadas con datos obtenidos en las pruebas estáticas a destrucción, ilustran los diferentes comportamientos de las tres vigas. Las características carga-flecha de las tres vigas resultaban casi idénticas desde la carga inicial hasta la rotura. Sin embargo, para cargas situadas más allá de la rotura las características de las flechas de las vigas dependían de la longitud de los revestimientos de los cables. La viga 1 flectó, para una carga dada después de rotura, menos que las vigas con cables revestidos.

\section{Rotura a flexión}

El número de grietas grandes por flexiơn en las vigas por efecto de la carga final indica también un efecto de los revestimientos. Como se muestra en la tabla 4, hay 18, 20 y 13 grietas grandes en las vigas 1,2 y 3 , respectivamente. Las grietas por flexión de la viga totalmente revestida, viga 3 , eran considerablemente más anchas para las cargas dichas que la de las otras dos vigas. Esto indica una adherencia diferente.

\begin{tabular}{|c|c|c|c|c|c|c|}
\hline \multirow{2}{*}{ Viga número } & \multicolumn{2}{|c|}{ MOMENTO FINAL } & \multirow{2}{*}{$\begin{array}{l}\text { Relación } \\
\frac{M_{\text {ens. }}}{M_{\text {calc. }}}\end{array}$} & \multirow{2}{*}{$\begin{array}{c}\text { Esfuerzo } \\
\text { máximo en el } \\
\text { hormigón } \\
\text { (luz media) }\end{array}$} & \multirow{2}{*}{$q$} & \multirow{2}{*}{$\begin{array}{l}\text { Número de las } \\
\text { grietas de fle- } \\
\text { xión mayores } \\
\text { al final del } \\
\text { experimento }\end{array}$} \\
\hline & $\begin{array}{l}\text { Ensayado } \\
(\mathrm{kg} \times \mathrm{m})\end{array}$ & $\begin{array}{l}\text { Calculado } \\
(\mathrm{kg} \times \mathrm{m})\end{array}$ & & & & \\
\hline 1, no revestida & 63.460 & 65.119 & 0,98 & 0,0038 & 0,058 & 18 \\
\hline 2, parcialmente revestida & 62.768 & 65.257 & 0,96 & 0,0032 & 0,054 & 20 \\
\hline 3 , totalmente revestida & 55.164 & 65.119 & 0,84 & 0,0009 & 0,055 & 13 \\
\hline
\end{tabular}

TABLA 4. CARACTERISTICAS DE LAS VIGAS 


\section{Deformaclón de los cables}

En la tabla 5 se aprecia la medida del incremento de la tensión en el cable debido a la carga de servicio durante el período de carga. No había evidencia de que estos esfuerzos hubieran roto las vigas, o provocado el deslizamiento de los cables, o producido un comportamiento anormal dentro de los límites de la carga de servicio, ya que las variaciones de tensión eran muy pequeñas.

TABLA 5. INCREMENTO DE ESFUERZOS EN LOS CABLES DE LA

CARA EXTERIOR, NIVEL INFERIOR, CON CARGA DE SERVICIO DURANTE EL PERIOdO DE CARga CIClica

\begin{tabular}{c|c|c|c} 
Ciclos & $\begin{array}{c}\text { Viga 1 } \\
\left(\mathrm{kg} / \mathrm{cm}^{2}\right)\end{array}$ & $\begin{array}{c}\text { Viga 2 } \\
\left(\mathrm{kg} / \mathrm{cm}^{2}\right)\end{array}$ & $\begin{array}{c}\text { Viga 3 } \\
\left(\mathrm{kg} / \mathrm{cm}^{2}\right)\end{array}$ \\
1 & 459,8 & & 459,8 \\
5 & 409,1 & 400,7 & 489,3 \\
$1 \times 10^{6}$ & 440,8 & 400,7 & 510,4 \\
$2,5 \times 10^{6}$ & 430,2 & 400,7 & 510,4 \\
$5 \times 10^{6}$ & 430,2 & 379,6 & -
\end{tabular}

Las típicas observaciones de tensión en los cables durante la carga estática a rotura pueden verse en la figura 10. Esta observación también diferencia claramente el comportamiento de las tres vigas. Se han elegido como típicos los datos de tensión de los cables del lado exterior en los planos inferior y medio. En el caso de la viga 1, la tensión del cable aumentó linealmente hasta que el hormigón se agrietó en las proximidades del medidor de deformaciones. Después del agrietamiento, la deformación creció hasta que el cable se rompió bajo la carga final cerca de la luz media. Este comportamiento fue típico de todos los cables en este espécimen.

El cable desnudo situado en el lado exterior del nivel medio de la viga 2 se comportó de un modo semejante al del cable de la viga 1 en el ensayo bajo carga estática. La tensión creció linealmente hasta la rotura $\mathrm{y}$, después de esto, en valores crecientes hasta el final. El cable revestido del lado exterior en el plano inferior de la viga 2 mostró comportamiento normal hasta un punto después de la carga de rotura. En un instante bien definido, la tensión empezó a decrecer y continuó así hasta que todos los cables se rompieron cerca de la luz media bajo la carga final. Hay que hacer notar que la tensión a $1,8 \mathrm{~m}$ de la luz media decreció poco y permaneció suficientemente por encima de la tensión inicial debida al pretensado.

En la viga 3, los cables exteriores de los niveles inferior y medio estaban revestidos. La relación tensióncarga indica claramente falta de adherencia en el tercer incremento después de producirse el agrietamiento del hormigón cerca del medidor de tensiones. Después de la pérdida de adherencia, la tensión del cable exterior en el nivel más bajo decreció muy por debajo de la tensión de pretensado.

Resumiendo, todos los cables revestidos sufrieron alguna pérdida de adherencia, circunstancia que no se produjo en los cables desnudos. Según fue aproximándose la carga final, el valor de la tensión de algunos cables de la viga totalmente revestida decreció muy por debajo del valor de pretensado inicial; en cambio, en el caso de la viga parcialmente revestida permaneció la bastante por encima del referido valor.

En todos los casos de decrecimiento imprevisto en la tensión, tales como los indicados en la figura 10, los cables que sobresalían por los extremos de las vigas empezaron a «introducirse» en la viga como ulterior demostración de la pérdida de adherencia inmediatamente después que las mediciones indicaron un decrecimiento de tensión. 


\section{Resulfados del ensayo: Vigas 4 y 5 Esfuerzo}

Los esfuerzos cortantes calculados y medidos en rotura para las vigas 4 y $\mathbf{5}$ se muestran en la figura 11 . Las fuerzas calculadas se determinaron por las actuales características de los materiales empleados a partir de la ecuación (26-10) de la sección 2610 de la 1963 ACI Code:

$$
A_{v}=\frac{\left(V_{u}-\Phi \cdot V_{c}\right) s}{\Phi \cdot d \cdot f_{y}}
$$

en donde:

$$
\begin{aligned}
A_{v} & =\text { área del refuerzo de nervio colocada perpendicularmente al eje del elemento; } \\
\Phi & =\text { factor de reducción de capacidad; } \\
V_{u} & =\text { cortante debido a la carga final determinada; } \\
V_{c} & =\text { cortante soportado por el hormigón; } \\
s & =\text { separación longitudinal del refuerzo de nervio; } \\
d & =\text { distancia de la fibra extrema de compresión al centroide de la fuerza de pretensado; } \\
f_{y} & =\text { rendimiento del esfuerzo del refuerzo no pretensado. }
\end{aligned}
$$

La expresión estaba readaptada y $V_{u}$ calculado usando $\Phi$ igual a la unidad, y $f_{y}=3.550,5 \mathrm{~kg} / \mathrm{cm}^{2}$. El cortante principal de rotura, $V_{\varepsilon}$, estaba calculado en la ecuación [3] para cada sección transversal por los procedimientos dados en la sección 2610 .

Los momentos finales de ambas vigas estaban calculados para la sección media empleando la fórmula [2], con $\Phi$ igual a la unidad, el esfuerzo en el cable igual al esfuerzo de tensión medido y el esfuerzo de compresión del hormigón igual a los valores medidos.

La viga 4 rompió en cortante horizontal a lo largo de la unión del nervio y de la cara inclinada del reborde inferior, como indica la figura 12 . El plano de rotura se extendía desde la región del punto de carga más exterior hasta el extremo de la viga. La rotura fue instantánea y tuvo lugar mientras la carga estaba siendo incrementada hasta el nivel siguiente. En la zona de rotura, la relación de $V_{\text {ens. a }}$ $V_{\text {cale. }}$ variaba de 0,88 a 0,92 . La relación en rotura de $M_{\text {ens. }}$ a $M_{\text {cale. }}(58.068 \mathrm{~kg} \times \mathrm{m} / 65.533 \mathrm{~kg} \times \mathrm{m})$ era 0,98 .

La viga 5 se dejó en flexión hasta que todos los cables rompieron en una sección próxima a la luz media, como indica la figura 13. Al final, la relación entre $M_{\text {ens. }}(64.427 \mathrm{~kg} \times \mathrm{m})$ y $M_{\text {cale. }}(63.736 \mathrm{~kg} \times \mathrm{m})$ era 1,01. En la región desde el punto de carga interior hasta el extremo de la viga la relación $V_{\text {ens. a }} V_{\text {cale. }}$ variaba de 0,84 a 1,01 , con lo que el esfuerzo cortante era disipado prácticamente.

\section{Relación carga-flecha}

Las curvas carga-flecha para la viga 4 (no revestida) y la viga 5 (parcialmente revestida) pueden apreciarse en la figura 9 y están comparadas con las vigas correspondientes en las series de flexión. Se ve que las vigas 2 y 5 señalan características casi idénticas y ambas presentaron la ductilidad típica en una rotura a flexión. Por otra parte, la viga 4 no cedió excesivamente. La capacidad a flexión de la viga 4 no fue alcanzada y sólo se reprodujo una parte de la curva carga-flecha de la viga 1.

\section{Rofura}

En ambas vigas la rotura se limitó, principalmente, a la zona comprendida entre los puntos de carga externos. Las grietas diagonales eran del mismo tipo que las grietas de flexión y se pudieron observar en el área limitada entre los puntos de carga interior y exterior. Al llegar la etapa en que las grietas diagonales se habían extendido al reborde superior de la viga premoldeada, empezaron a formarse grietas horizontales en la unión del nervio con la cara inclinada del reborde inferior. En ambas vigas, justo antes de la rotura, había grietas horizontales continuas desde el punto de carga interior hasta el exterior a ambos lados de la luz media. La grieta tipo de la viga 5 era muy parecida a la de viga 4 , seña- 
lando que la capacidad de cortante estaba casi agotada cuando se rompieron los cables. No se observaron grietas diagonales en el tramo cercano a los apoyos en ninguna de las dos vigas. Esto se justificaba por las lecturas de tensiones medidas desde los estribos.

\section{Tensiones en los cables}

El cable en la viga 4 no indicaba pérdida evidente de adherencia en la rotura a cortante del espécimen. La tensión del cable crecía al final del experimento de forma semejante a la señalada en la figura 10 para el cable $A$ de las vigas 1 y 2 . Las lecturas de los medidores de cuadrante en los extremos de las vigas no indicaban movimientos diferenciales entre el extremo del cable y el hormigón.

En la viga 5, los cuatro cables revestidos mostraban algún internamiento en los extremos de las vigas. Los dos cables exteriores de la hilera del fondo del refuerzo indicaban un deslizamiento considerable dentro de la viga. Los otros dos cables con revestimientos más largos tuvieron sustancialmente movimientos más cortos. Excepto para la situación medida cerca de la sección de rotura, todas las tensiones de los cables crecieron continuamente al final del experimento. Las lecturas de tensión desde la línea de medidores contigua al plano de rotura se hicieron erróneas, y en ambas las tensiones del cable crecían y decrecían mientras se aproximaba la carga final a flexión. Los efectos locales debidos a la proximidad del plano de rotura eran responsables en apariencia de la producción de medidas erróneas.

\section{Conclusiones}

Los tres ensayos de vigas $\mathrm{T}$ utilizando cargas dinámicas no demostraban efectos perjudiciales del revestimiento del cable en elementos pretensados sometidos a $5 \times 10^{6}$ ciclos bajo carga de servicio.

Después de la carga de rotura, y bajo cargas estáticas, ocurrió alguna pérdida de adherencia en todos los cables revestidos.

Los resultados de los dos ensayos en que las vigas tenían un esfuerzo menor que el cortante requerido, indican que el revestimiento no produce efectos perjudiciales sobre el esfuerzo cortante.

Hay evidencia de que el requisito de la 1963 ACI Building Code (ACI 318-63) (3) sobre la longitud de empotramiento del cable, en relación con la adherencia del mismo, en su sección 2611, no puede aplicarse directamente al cable revestido. Sin embargo, el comportamiento en estas pruebas investigadoras de vigas de cables revestidos con longitudes de empotramientos dobles que las requeridas por la sección 2611, coincidió por completo con el comportamiento a flexión de una viga pretensada semejante con los cables sin revestir en absoluto.

\section{Reconocimiento}

La investigación cuya exposición se acaba de hacer tuvo lugar en el Structural Laboratory of the Portland Cement Association, bajo la dirección del Dr. Eivind Hognestad, cooperando en la misma varios de sus miembros. Mención especial se debe a B. W. Fullhart, O. A. Kurvits, R. K. Richter, y A. G. Aabey, por su contribución durante el experimento.

Este artículo ha sido publicado con la autorización del Prestressed Concrete Institute.

\section{Bibliografía}

(1) KAAR, P. H.; LAFraUgh, R. W., and MASS, M. A.: "Influence of Concrete Strength on Strand Transfer Length", Journal of the Prestressed Concrete Institute, Vol. 8, N. ${ }^{\circ}$, octubre 1963, pp. 47-67 (PCA Development Department Bulletin D71).

(2) Hanson, N. W., and KAAR, P. H.: "Flexural Bond Tests of Pretensioned Prestressed Beams", Journal of the American Concrete Institute, Proceedings, Vol. 55, enero 1959, pp. 783-802 (PCA Development Department Bulletin D28). 
(4) ACI Standard ACI 318-63, Building Code Requirements for Reinforced Concrete, American Concrete Institute, 1963.

(4) Standard Specifications for Highway Bridges, 8th Edition, American Association of State Highway Officials, 1961.

(5) Hornestad, E.; Hanson, N. W.; Kritz, L. B., and KuRvis, O. A.: "Facilities and Test Methods of the PCA Structural Laboratory", under various titles in Journal of the PCA Research and Development Laboratories, Vol. 1, N. ${ }^{\circ}$, enero 1959 , pp. 12-20, 40-44; Vol. 1, N. 2 , mayo 1959, pp. 30-37; Vol. 1, N. ${ }^{\circ} 3$, septiembre 1959, pp. 35-41 (PCA Development Department Bulletin D33). Also: Hanson, N. W.; Kurvits, O. A., and MATrock, A. H.: "Semi-Automatic Processing of Strain and Load Measurements", Journal of the PCA Research and Development Laboratories, Vol. 3, N. 2, mayo 1961, pp. 27-31.

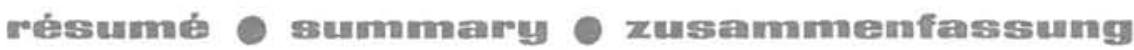

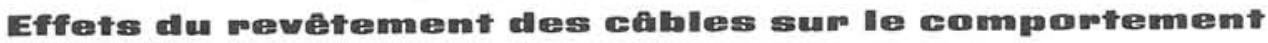 diles pountres préćom trmaimines}

Paul H. Kaar et Donald D. Magura, ingénieurs

Il s'agit ici d'une étude sur le revêtement en tubes de matière plastique, employés pour prévenir l'adhérence entre le câble et le béton près des extrémités des poutres précontraintes. Des essais effectués sur cinq poutres $\mathrm{T}$ de $10,3 \mathrm{~m}$ de longueur ont permis de constater que la longueur d'encastrement exigée par la section 2611 de la 1963 ACI Building Code n'est pas appropriée dans le cas de câbles revêtus. La longueur nécessaire pour permettre la flexión maximale
et l'effort tranchant dans les poutres, lors de l'emploi du revêtement, est double de la longueur de l'ancrage signalée par cette norme.

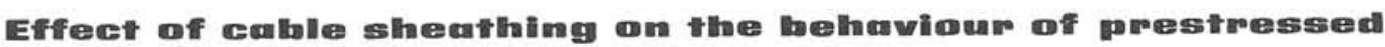 bearims}

Paul H. Kaar and Donald D. Magura, engineers

This deals with an investigation of plastic sheathing applied to prestressing cables near the end of the beams, to prevent their direct bond with the concrete. Tests on five $T$ beams, of $10.3 \mathrm{~m}$ length showed that the length of bonding required by section 2611 of the 1963 ACI Building Code is inadequate in the case of sheathed cables. The necessary length so that maximum bending moment and shear force can take place, when sheathing is adopted, is twice the anchorage length that the above specification requires.

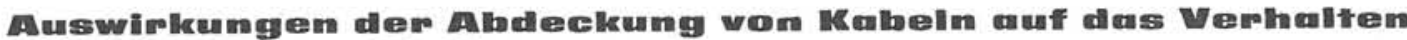

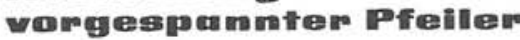

Paul H. Kaar und Donald D. Magura, Ingenieure

Es handelt sich hier um eine Studie über die Verkleidung mit Plastikrohren zum Schutz gegen Adhäsion zwischen Kabel und Beton in der Nähe der Enden der vorgespannten Pfeiler. Die Versuche mit fünf T.Pfeilern von 10,3 m Länge zeigten, dass die Einspannungslänge, die Abschnitt 2611 ACI Building Code für verkleidete Kabel angibt, nicht zutreffend ist. Die zur Entwicklung der höchsten Biegung und Scherkraft in den Pfeilern notwendige Länge ist bei Verkleidung doppelt so gross wie die Verankerungslänge die die erwähnte Norm angibt. 